\title{
Near-Band-Edge Photoluminescence from Very High Quality Hexagonal ZnO Bulk Crystals
}

\author{
N.T.T. LIEU, D.H. DAT AND N.Q. LIEM* \\ Institute of Materials Science, NCST of Vietnam \\ 18 Hoang Quoc Viet - Cau Giay - Hanoi, Vietnam
}

(Received September 24, 2002)

\begin{abstract}
The near-band-edge photoluminescence spectra of very high quality hexagonal $\mathrm{ZnO}$ single crystals in the temperature range between 9 and $305 \mathrm{~K}$ were measured. Based on the energetic positions and the evolutions of well-resolved photoluminescence lines with temperature and with excitation power density we interpret the observed photoluminescence lines as resulting from recombination of the free-exciton, bound-exciton, biexciton, inelastic exciton-exciton collision and electron-hole plasma.
\end{abstract}

PACS numbers: 71.35.-y, 78.55.-m, 78.55.Et

\section{Introduction}

In the last years, $\mathrm{ZnO}$ has been paid much attention because of its advantages: the direct electronic transition, the very high free-exciton binding energy $(60 \mathrm{meV})$, the large band-gap energy, etc. The behaviours mentioned above make $\mathrm{ZnO}$ suitable for exciton-related optoelectronic devices working in the short wavelength range and at room temperature [1-5]. Furthermore, $\mathrm{ZnO}$ doped with transition metals demonstrates to be very promising diluted magnetic semiconductor for spintronic devices with the Curie temperature higher than the room temperature [6-8]. Achievements in the materials preparation as well as in the physics understandings of $\mathrm{ZnO}$ have been obtained ${ }^{\dagger}[1-11]$. However, the variations in quality of

* corresponding author; e-mail: nqliem@ims.ncst.ac.vn

${ }^{\dagger}$ At the Tenth International Conference on II-VI Compounds held in Bremen, Germany, 9-14 September 2001, $\mathrm{ZnO}$ was extensively discussed in a separate section with numerous papers of $12 \%$ of the total papers presented. 
materials prepared and different experimental techniques used have given rise to the discrepancy in reported data for the near-band-edge photoluminescence (PL) from $\mathrm{ZnO}$ crystals. Practically, it is hard to observe the PL from free-exciton recombination in single crystals because of the crystalline quality and the resonant reabsorption process. Moreover, since Bohr radius of the free exciton in $\mathrm{ZnO}$ is small $(18 \AA)$, it is expected that the critical concentration of excitons is high. As a consequence, strong interactions between exciton and exciton and other particles or quasi-particles take place that make the near-band-edge PL spectra complex. In other words, all exciton-related entities such as the free excitons, bound excitons, biexcitons, etc. and the free carriers compete together and contribute to the emission at the same time. There is the fact that in many published papers the $\mathrm{PL}$ spectra from $\mathrm{ZnO}$ were presented very low in resolution and different in spectral feature consequent upon the different samples prepared and experiments used. These obstructed the clear-cut discussion on the emission lines in $\mathrm{ZnO}$ crystals.

In this paper, we present the PL properties of very high quality hexagonal $\mathrm{ZnO}$ single crystals in the temperature range between 9 and $305 \mathrm{~K}$. At low temperature the PL spectra were obtained in very high resolution that consist of the various emission lines resulting from recombinations of the exciton and exciton-related entities. Thermal quenching of the emission lines as well as their evolutions with excitation power densities were systematically studied that enabled us to interpret the radiative mechanism, to determine exactly the peak energy corresponding to each electronic transition, and to observe transformations between the mentioned emission lines.

\section{Experimental}

The samples used in our study are $\mathrm{ZnO}$ single crystals grown by a vapour-phase method. These crystals have the hexagonal structure with mirror-like crystalline facets. In the PL measurements the samples were mounted on the cold finger of a closed-cycle helium cryostat (APD Cryogenics Inc. Model HC-2). Sample temperature was maintained at any required value from 9 to $305 \mathrm{~K}$ by using a temperature controller (LakeShore 330). Low-pressure nitrogen gas laser (repetition of $10 \mathrm{~Hz}$, pulse width of $7 \mathrm{~ns}$ ) was used as the excitation source. The use of pulsed-laser excitation in combination with an appropriate electronic treatment unit gave a way for the PL measurement with moderate excitation power densities in the integrated regime. Spectral resolution was better than $0.3 \mathrm{meV}$ achieved by using a double monochromator with two $1200 \mathrm{Gr} / \mathrm{mm}$ gratings (Jobin-Yvon HRD1). The PL signal then was detected by a photomultiplier tube (FEU-100) and averaged in multi-pulses at each spectral point by a $1.5 \mathrm{GHz}$ digital oscilloscope (LeCroy 9362) for improving the signal-to-noise ratio. 


\section{Results and discussion}

Figure 1 shows the PL spectra of a $\mathrm{ZnO}$ single crystal at $9 \mathrm{~K}$. The excitation power density was chosen to be of $10 \mathrm{~kW} / \mathrm{cm}^{2}$ for the reason to limit interactions between excitons and other particles. The inset shows the very near-band-edge PL lines. We like noting that the very narrow emission lines (full width at half maximum, FWHM,$<3 \mathrm{meV}$ ) obtained are the evidence for the high crystalline quality of the samples under study. Because the spectral resolution of the PL measurement was set to be better than $0.3 \mathrm{meV}$ thus the emission lines obtained are well-resolved by self-sample and the affection of instrumental response to the overall resolution could be ignored. The emission lines are labeled $\mathrm{X}_{\mathrm{L}}$ at $3.375 \mathrm{eV}, \mathrm{D}^{0} \mathrm{X}$ at $3.360 \mathrm{eV}$, and $\mathrm{XX}$ at $3.357 \mathrm{eV}$. The assigned labels imply that the corresponding PL lines are the indecisively free-excitonic at low energy, neutral donor bound excitonic, and biexcitonic transitions, respectively [12]. We mention the indecisive feature of the $\mathrm{X}_{\mathrm{L}}$ emission line because of the large discrepancy in reported data for the free-excitonic and biexcitonic emissions. It is therefore necessary to discuss the $\mathrm{X}_{\mathrm{L}}$ and XX emission lines in more detail. For the biexcitonic emission (the XX line) in $\mathrm{ZnO}$ single crystals, we clearly observed it under certain excitation conditions. Its characteristics including the temperature-dependent PL will be reported in a separate paper.

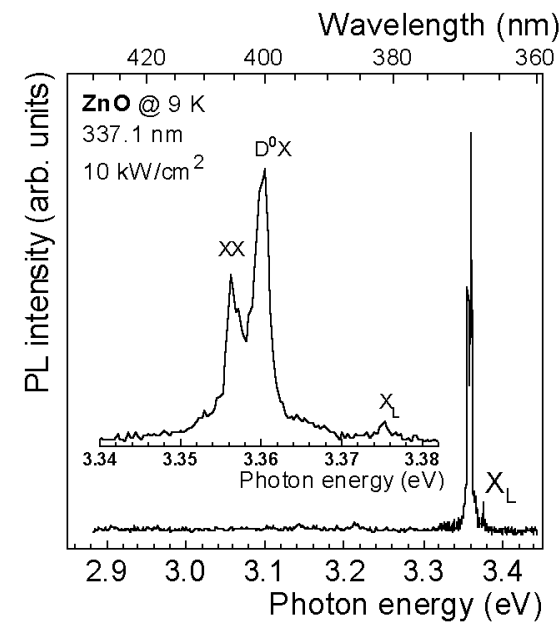

Fig. 1. PL spectra of $\mathrm{ZnO}$ bulk crystals at $9 \mathrm{~K}$ with an excitation power density of $10 \mathrm{~kW} / \mathrm{cm}^{2}$. The inset shows an expansion of the excitonic part for clarity.

We now focus on the $\mathrm{X}_{\mathrm{L}}$ emission line. In the literature, the PL line around $3.375 \mathrm{eV}$, i.e. the one corresponds to the $\mathrm{X}_{\mathrm{L}}$ line in this report, has been widely interpreted as resulting from the free-excitonic transition. In fact, the peak energy of the mentioned emission in the various kinds of $\mathrm{ZnO}$ reported are somewhat varied 


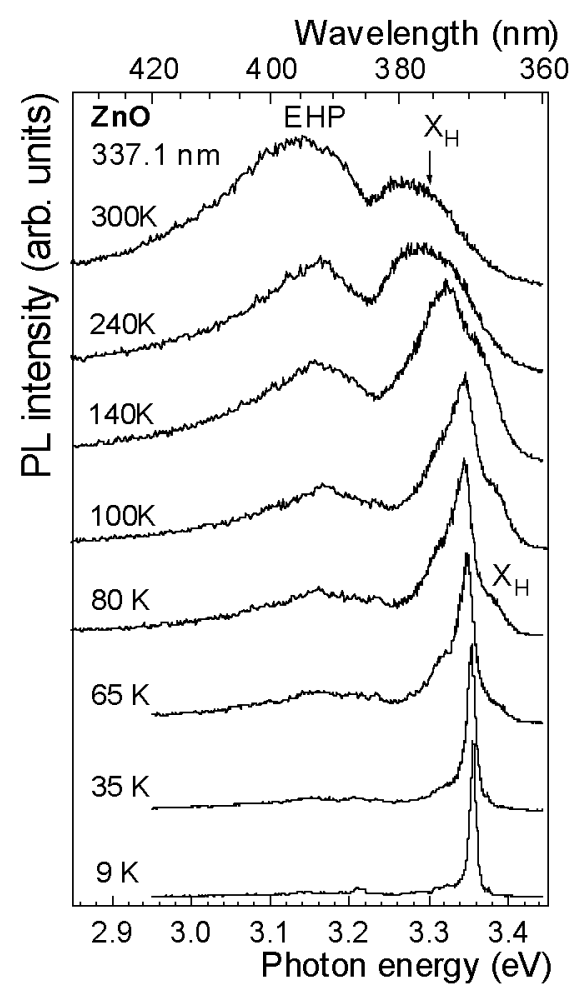

Fig. 2. The evolution of $\mathrm{PL}$ from $\mathrm{ZnO}$ bulk crystals as a function of temperature with an excitation power density of $1 \mathrm{MW} / \mathrm{cm}^{2}$.

[1-3, 9-13]. This may originate from different kinds of samples and may originate from unreasonable assignments as well. We observed several features, which are not characteristic of the free-excitonic transition, from the so-called free-exciton emission line at $3.375 \mathrm{eV}$. In Fig. 2 the evolution of PL spectra from a $\mathrm{ZnO}$ single crystal as a function of temperature is shown. The excitation power density was chosen to be $\approx 1 \mathrm{MW} / \mathrm{cm}^{2}$. At this excitation power density, the $\mathrm{D}^{0} \mathrm{X}$ emission was almost intensity-saturated hence a minor contribution of this to the overall spectra needs to be considered even at $9 \mathrm{~K}$. The first interesting result is that the $\mathrm{X}_{\mathrm{L}}$ emission line was very fast thermally quenched. $\mathrm{At} \approx 50 \mathrm{~K}$, no trace of the $\mathrm{X}_{\mathrm{L}}$ line remained although the free-exciton binding energy of $\mathrm{ZnO}$ is $60 \mathrm{meV}$, which corresponds to the thermal quenching at about $700 \mathrm{~K}$. Moreover, along with the disappearance of the $\mathrm{X}_{\mathrm{L}}$ line a new PL line labeled $\mathrm{X}_{\mathrm{H}}$ appears at the higher energy side and its relative intensity is increased gradually as compared to those of the other PL lines. We could determine the exact energy of the $\mathrm{X}_{\mathrm{H}}$ line by a curve fitting procedure [14], which was performed on the spectrum measured at low excitation power density (in order to decrease the error causing by overlap- 
ping the various emission lines). The energy of the $\mathrm{X}_{\mathrm{H}}$ line is determined to be $3.384 \mathrm{eV}$ at $80 \mathrm{~K}$ and $3.315 \mathrm{eV}$ at $305 \mathrm{~K}$. The $\mathrm{X}_{\mathrm{H}}$ emission line developed with temperature over the other band-edge emission lines and became dominant at room temperature. The $\mathrm{X}_{\mathrm{H}}$ line at higher energy than that of the $\mathrm{X}_{\mathrm{L}}$ line and its dominance at room-temperature spectra indicate reasonably the characteristics of the free-exciton emission in $\mathrm{ZnO}$ crystal. Using the binding energy of the free exciton in $\mathrm{ZnO}$ crystal of $60 \mathrm{meV}$, the band-gap energy of the hexagonal ZnO crystal is determined to be $3.375 \mathrm{eV}$ at room temperature. This result is in very good agreement with that obtained by the optical absorption measurement [15]. Generally, it is very difficult to observe the free-exciton emission because of the crystal quality: a small amount of impurities (donors or acceptors) or defects can act as traps. For those reasons, recombination of the electrons and holes would take place at the binding centres indicated above. Consequently, in crystals we usually observe the emission of bound excitons. On the other hand, because of the resonance in energy, the emission from free excitons recombination can highly be reabsorbed. Checking further in behaviour of the $\mathrm{X}_{\mathrm{L}}$ line one can see that it has the very narrow FWHM. This is the character somewhat related to the binding state but not to the free-exciton state. In the case of $\mathrm{ZnO}$ crystals, the critical concentration of free exciton is very high due to its small Bohr radius that make the interaction between excitons strong. As a result, the broadening of the free-exciton PL line is indispensable and significant. From deconvolution of the $80 \mathrm{~K} \mathrm{PL}$ spectrum we obtained the FWHM of the $\mathrm{X}_{\mathrm{H}}$ line to be reasonable of $25 \mathrm{meV}$.

With increasing temperature the dissociation of the biexcitons and bound excitons takes place first, which gives rise to the decrease in the biexciton and bound-exciton PL intensities, respectively. The PL from recombination of the free excitons can be increased because the populations of the free excitons increased. The increase in the free-exciton population and the increase in exciton-exciton scattering rate with temperature also directly give rise to the increase in the emission intensity resulting from exciton-exciton collisions. Moreover, the increase in scattering between the particles makes the dissociation of the excitons increasable, and hence the density of the free carriers increased, too. Consequently, the PL band due to electron-hole recombination in the plasma state (the peak position of the electron-hole plasma (EHP) band was at about $3.15 \mathrm{eV}$ ) was increased much with temperature. In principle, at the thermodynamic equilibrium the increase or decrease in the PL intensities can be expressed by the set of the equations describing the dependencies of the electron and hole populations on the energy and sample temperature.

With increase in excitation power density, the evolutions of PL intensities corresponding to the various transitions are different: $\mathrm{D}^{0} \mathrm{X}$ line related to a finite number of donors and it is therefore intensity-saturated, while the PL intensity of the XX line became much stronger than other lines. The predominant increase in the PL intensity of the XX line with increasing excitation power density is very 


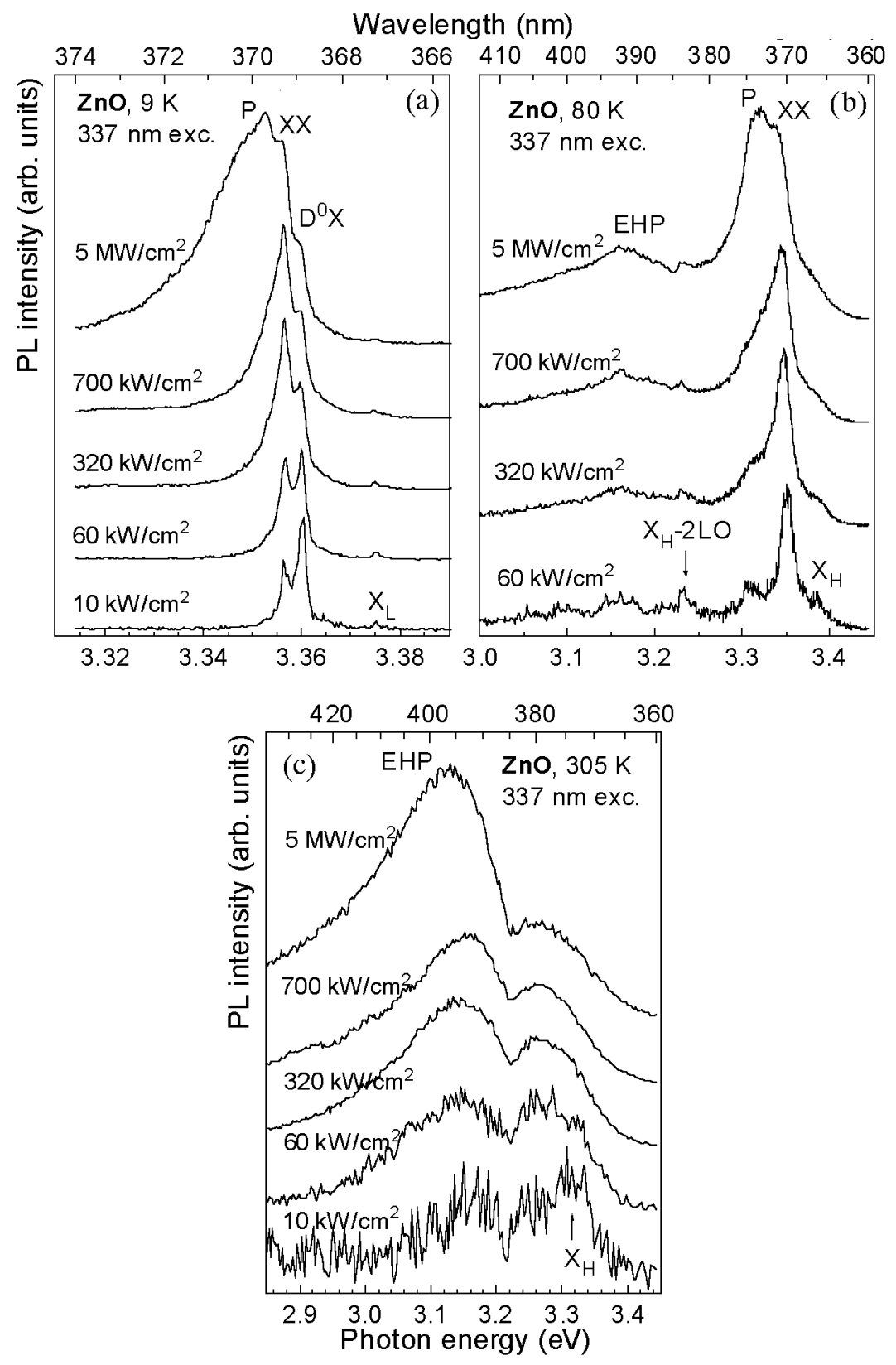

Fig. 3. PL spectra of $\mathrm{ZnO}$ bulk crystals at (a) 9, (b) 80 and (c) $305 \mathrm{~K}$ as functions of excitation power densities.

characteristic of the biexcitonic luminescence. On the other hand, the intensity of the $\mathrm{P}$ line was gradually increased and became a pronounced band (see Figs. 3a 
and b). At the excitation power density over $1 \mathrm{MW} / \mathrm{cm}^{2}$ the $\mathrm{P}$ emission is the predominant component in the whole spectrum with a shape of broadband tailing to the lower energy side (see the curves corresponding to the $5 \mathrm{MW} / \mathrm{cm}^{2}$ excitation power density). The $\mathrm{P}$ band observed in the spectrum is composed by the superposition of the $\mathrm{P}_{n}$ emission lines $(n=2 \div \infty)$, which were formed by electron-hole recombination during the inelastic exciton-exciton collision. The energy of the emitted photons is expressed as [16]:

$$
E_{n}=E_{\mathrm{x}}-E_{\mathrm{x}}^{\mathrm{b}}\left(1-\frac{1}{n^{2}}\right)-\frac{3}{2} k T,
$$

where $E_{\mathrm{x}}$ is the free-exciton energy, $E_{\mathrm{x}}^{\mathrm{b}}$ the binding energy of free exciton, and the last term expresses the thermodynamic energy of carriers. The expression shows a separation of the $\mathrm{P}_{2}$ line from the left $\mathrm{P}_{3 \rightarrow \infty}$ lines, which appear as a continuum band composed by overlapping of them. In fact, the superposition of all $\mathrm{P}_{2}$ (with more intense intensity because of higher electron-hole recombination rate) and $\mathrm{P}_{3 \rightarrow \infty}$ lines results in the very asymmetric $\mathrm{P}$ band peaking close to the peak of the $\mathrm{P}_{2}$ emission line. Here, a problem comes out: if the free-exciton energy $E_{\mathrm{x}}$ is not exactly determined, one cannot determine $E_{n}$. In our experiment, $E_{2}$ is extracted to be $3.346 \mathrm{eV}$ at $9 \mathrm{~K}$ corresponding to $E_{\mathrm{x}}$ of $3.391 \mathrm{eV}$, which is very far from reported data for the so-called free exciton $\mathrm{A}$ at $3.375 \mathrm{eV}$. However, this value is very close to the so-called free exciton $B$ in numerous publications [17-19]. The higher energy $E_{\mathrm{x}}$ of the free exciton is again ruling out the free-excitonic nature of the $3.375 \mathrm{eV}$ emission line. It is very nice that an extrapolation by the least-squares fit of the $\mathrm{X}_{\mathrm{H}}$ emission energy as a function of temperature to Varshni's equation give the same value for $\mathrm{X}_{\mathrm{H}}$ at $9 \mathrm{~K}$, namely $3.391 \mathrm{eV}$ [20]. This is a good support to the true free-exciton transition be responsible to the $\mathrm{X}_{\mathrm{H}}$ emission in $\mathrm{ZnO}$.

Since the biexcitonic luminescence line was nicely recorded, its binding energy is therefore exactly determined to be $34 \mathrm{meV}$ if the $3.391 \mathrm{eV}$ emission line is considered to originate from the free-excitonic transition. The value obtained is different from reported data: much higher than $14.7 \mathrm{meV}$ in Ref. [2]. This is because of the following reasons. First, in our opinion, the accuracy of reported data is not appropriate since they were deduced from the unresolved absorption and/or photoluminescence spectra. Second, the most important reason is that the so-called free-excitonic transition was not exactly identified as we have just proved above. In our experiments, the high-resolution PL spectra were recorded on the very high quality $\mathrm{ZnO}$ single crystals that enabled us to determine exactly the energy of the spectral lines. Here, we would like to note that the $3.375 \mathrm{eV}$ emission line may correspond to a localized state which strongly relates to the free-exciton and biexciton recombinations. In fact, the population evolving with temperature of each quasi-particle like exciton and/or biexciton etc. and the thermodynamic population of them at a certain temperature are very complicated.

At room temperature or higher, the EHP emission intensity is much increased with increasing excitation density (Fig. 3c). This is the main reason for 
that the laser light from $\mathrm{ZnO}$ material originates from electron-hole recombination in the plasma states, i.e. from the EHP band $[1,10]$. Analyzing in detail the temperature-dependent PL spectra which are represented in Fig. 2 and the excitation power density-dependent PL spectra in Fig. 3, we see that with increasing sample temperature or excitation power density the peak position of the EHP band shifted toward the lower energy side. However, the physical mechanisms governing the two processes of the energy redshift are different. With increasing temperature the band gap of $\mathrm{ZnO}$ crystals is narrowed due to the creation of microfields induced by lattice heat. There are two main contributions to the temperature dependence of the band-gap energy in semiconductors, namely the electron-phonon interaction and the thermal dilatation of crystalline lattice [14, 21], thus the energy shift of the EHP band takes place accordingly. The increase in carrier density with the photoexcitation density causes another effect (the band-gap renormalization) that give rise to the reduction of the band-gap energy. Consequently, the exciton PL lines and the EHP band were shifted towards the lower energy side. In the case of very high carrier density it is necessary to study the screening effect, which causes the shift of the exciton emission lines towards the higher energy side. However, in the concrete case of $\mathrm{ZnO}$ crystals the shift of the near-band-edge PL lines towards the lower energy side is predominant because of the band-gap renormalization [22]. In our experiments, we observed clearly the band-gap renormalization at fixed sample temperatures by a variation in excitation power density.

\section{Conclusion}

In conclusion, the high resolution PL measurements were performed on the very high quality $\mathrm{ZnO}$ single crystals. The detailed results obtained from the evolution of the PL with excitation power density and with sample temperature enable us to interpret exactly the free-exciton, bound-exciton, biexciton transitions, etc. The true free-exciton transition is definitely confirmed and its energy at different temperatures ranging from 9 to $305 \mathrm{~K}$ was exactly determined by the PL measurement, and that also means the band-gap energy of $\mathrm{ZnO}$ at corresponding temperatures was exactly determined. Thermodynamic processes corresponding to the thermal quenching of the bound and localized excitonic emissions to generate the free excitons at higher temperatures as well as the repopulation between them in thermodynamic equilibriums at certain excitation power density and/or sample temperature were briefly discussed.

\section{Acknowledgments}

The authors thank Prof. Tadashi Itoh for helpful discussions. Support from the National Programme for Basic Research of Vietnam is gratefully acknowledged. 


\section{References}

[1] A. Yamamoto, T. Kido, T. Goto, Y. Chen, T. Yao, A. Kasuya, Appl. Phys. Lett. 75, 469 (1999).

[2] J.M. Hvam, G. Blattner, M. Reuscher, C. Klingshirn, Phys. Status Solidi B 118, 179 (1983).

[3] Y. Chen, N.T. Tuan, Y. Segawa, H. Ko, S. Hong, T. Yao, Appl. Phys. Lett. 78, 1469 (2001).

[4] A. Ohtomo, K. Tamura, M. Kawasaki, T. Makino, Y. Segawa, Z.K. Tang, G.K.L. Wong, Y. Matsumoto, H. Koinuma, Appl. Phys. Lett. 77, 2204 (2000).

[5] D.M. Bagnall, Y.F. Chen, Z. Zhu, T. Yao, M.Y. Shen, T. Goto, Appl. Phys. Lett. 73, 1038 (1998).

[6] K. Ueda, H. Tabata, T. Kawai, Appl. Phys. Lett. 79, 988 (2001).

[7] T. Dietl, M. Sawicki, L.V. Khoi, J. Jaroszynski, P. Kossacki, J. Cibert, D. Ferrand, S. Tatarenko, A. Wasiela, Phys. Status Solidi B 229, 665 (2002).

[8] K. Sato, H. Katayama-Yoshida, Semicond. Sci. Technol. 17, 367 (2002).

[9] C. Klingshirn, Phys. Status Solidi B 71, 547 (1975).

[10] Y. Sun, J.B. Ketterson, G.K.L. Wong, Appl. Phys. Lett. 77, 2322 (2000).

[11] N. Ohashi, T. Sekiguchi, K. Aoyama, T. Ohgaki, Y. Terada, I. Sakaguchi, T. Tsurumi, H. Haneda, J. Appl. Phys. 91, 3658 (2002).

[12] N.T.T. Lieu, N.Q. Liem, D.X. Thanh, Proc. Fifth Vietnamese-German Seminar on Physics and Engineering, Hue 2002(Tu-99), in press.

[14] H.D. Sun, Y. Segawa, M. Kawasaki, A. Ohtomo, K. Tamura, H. Koinuma, J. Appl. Phys. 91, 6457 (2002).

[14] Nguyen Quang Liem, Vu Xuan Quang, Do Xuan Thanh, Joo In Lee, Dongho Kim, Solid State Commun. 117, 255 (2001).

[15] V. Srikant, D.R. Clarke, J. Appl. Phys. 83, 5447 (1998).

[16] C. Klingshirn, H. Haug, Phys. Rep. 70, 315 (1981).

[17] J. Lagois, K. Hummer, Phys. Status Solidi B 72, 393 (1975).

[18] M. Fiebig, D. Frohlich, Ch. Pahlke-Lerch, Phys. Status Solidi B 177, 187 (1993).

[19] S.F. Chichibu, A. Tsukazaki, M. Kawasaki, K. Tamura, Y. Segawa, T. Sota, H. Koinuma, Appl. Phys. Lett. 80, 2860 (2002).

[20] N.Q. Liem, N.T.T. Lieu, D.X. Thanh, to be published in Solid State Commun., 2002.

[21] W.W. Piper, D.T.F. Marple, P.D. Johnson, Phys. Rev. 110, 323 (1958).

[22] D.C. Reynolds, D.C. Look, B. Jogai, J. Appl. Phys. 88, 5760 (2000). 